\title{
Investigation into multiaxial fatigue character of thermomechanical damage on high-speed railway brake disc
}

\author{
Chun $\mathrm{Lu}^{1}$, Jiliang $\mathrm{Mo}^{1}$, Ruixue $\mathrm{Sun}^{1}$, Yuanke $\mathrm{Wu}^{1}$, Zhiyong $\mathrm{Fan}^{1}$, and Zhongrong Zhou ${ }^{1}$ \\ ${ }^{1}$ Southwest Jiaotong University
}

October 14, 2020

\begin{abstract}
The multiaxial fatigue character of high-speed railway brake disc thermomechanical damage is studied in this work. Although the amplitude and distribution of temperature, strain and stress are similar with uniform and rotation loading methods, the multiaxial behavior and out-of-phase failure status can only be revealed by the latter one. With the help of a multiaxial fatigue model, fatigue damage evaluation and fatigue life prediction are implemented, the contribution of uniaxial fatigue parameter, multiaxial fatigue parameter and out-of-phase failure parameter to the total damage is discussed, the damage will be underestimated without the consideration of multiaxial characters and out-of-phase failure status. It can be announced that brake disc fatigue belongs to the type of multiaxial thermomechanical fatigue. The results of this work can help to understand the damage mechanism of high-speed railway brake disc, and provide theoretical foundation for design optimization from the perspective of multiaxial thermomechanical fatigue resistance.
\end{abstract}

\section{Hosted file}

main document.pdf available at https://authorea.com/users/367130/articles/486664investigation-into-multiaxial-fatigue-character-of-thermomechanical-damage-on-highspeed-railway-brake-disc

\section{Hosted file}

Table 1.pdf available at https://authorea.com/users/367130/articles/486664-investigationinto-multiaxial-fatigue-character-of-thermomechanical-damage-on-high-speed-railwaybrake-disc

\section{Hosted file}

Table 2.pdf available at https://authorea.com/users/367130/articles/486664-investigationinto-multiaxial-fatigue-character-of-thermomechanical-damage-on-high-speed-railwaybrake-disc

\section{Hosted file}

Table 3.pdf available at https://authorea.com/users/367130/articles/486664-investigationinto-multiaxial-fatigue-character-of-thermomechanical-damage-on-high-speed-railwaybrake-disc

\section{Hosted file}

Figure 1.pdf available at https://authorea.com/users/367130/articles/486664-investigationinto-multiaxial-fatigue-character-of-thermomechanical-damage-on-high-speed-railwaybrake-disc 


\section{Hosted file}

Figure 2.pdf available at https://authorea.com/users/367130/articles/486664-investigationinto-multiaxial-fatigue-character-of-thermomechanical-damage-on-high-speed-railwaybrake-disc

\section{Hosted file}

Figure 3.pdf available at https://authorea.com/users/367130/articles/486664-investigationinto-multiaxial-fatigue-character-of-thermomechanical-damage-on-high-speed-railwaybrake-disc

\section{Hosted file}

Figure 4.pdf available at https://authorea.com/users/367130/articles/486664-investigationinto-multiaxial-fatigue-character-of-thermomechanical-damage-on-high-speed-railwaybrake-disc

\section{Hosted file}

Figure 5.pdf available at https://authorea.com/users/367130/articles/486664-investigationinto-multiaxial-fatigue-character-of-thermomechanical-damage-on-high-speed-railwaybrake-disc

\section{Hosted file}

Figure 6.pdf available at https://authorea.com/users/367130/articles/486664-investigationinto-multiaxial-fatigue-character-of-thermomechanical-damage-on-high-speed-railwaybrake-disc

\section{Hosted file}

Figure 7.pdf available at https://authorea.com/users/367130/articles/486664-investigationinto-multiaxial-fatigue-character-of-thermomechanical-damage-on-high-speed-railwaybrake-disc

\section{Hosted file}

Figure 8.pdf available at https://authorea.com/users/367130/articles/486664-investigationinto-multiaxial-fatigue-character-of-thermomechanical-damage-on-high-speed-railwaybrake-disc

\section{Hosted file}

Figure 9.pdf available at https://authorea.com/users/367130/articles/486664-investigationinto-multiaxial-fatigue-character-of-thermomechanical-damage-on-high-speed-railwaybrake-disc

\section{Hosted file}

Figure 10.pdf available at https://authorea.com/users/367130/articles/486664-investigationinto-multiaxial-fatigue-character-of-thermomechanical-damage-on-high-speed-railwaybrake-disc

\section{Hosted file}

Figure 11.pdf available at https://authorea.com/users/367130/articles/486664-investigationinto-multiaxial-fatigue-character-of-thermomechanical-damage-on-high-speed-railwaybrake-disc

\section{Hosted file}


Figure 12.pdf available at https://authorea.com/users/367130/articles/486664-investigationinto-multiaxial-fatigue-character-of-thermomechanical-damage-on-high-speed-railwaybrake-disc 